\title{
Next-to-Next-to-Leading Order Event Generation for Top-Quark Pair Production
}

\author{
Javier Mazzitelli, ${ }^{1}$ Pier Francesco Monni, ${ }^{2}$ Paolo Nason $\odot,{ }^{3}$ Emanuele Re $\odot,{ }^{4}$ Marius Wiesemann $\odot,{ }^{1}$ and Giulia Zanderighi ${ }^{1}$ \\ ${ }^{1}$ Max-Planck-Institut für Physik, Föhringer Ring 6, 80805 München, Germany \\ ${ }^{2}$ CERN, Theoretical Physics Department, CH-1211 Geneva 23, Switzerland \\ ${ }^{3}$ Università di Milano Bicocca and INFN, Sezione di Milano Bicocca, Piazza della Scienza 3, 20126 Milano, Italy \\ ${ }^{4}$ LAPTh, Université Grenoble Alpes, Université Savoie Mont Blanc, CNRS, 74940 Annecy, France
}

(Received 14 January 2021; accepted 27 May 2021; published 2 August 2021)

\begin{abstract}
The production of top-quark pairs in hadronic collisions is among the most important reactions in modern particle physics phenomenology and constitutes an instrumental avenue to study the properties of the heaviest quark observed in nature. The analysis of this process at the Large Hadron Collider relies heavily on Monte Carlo simulations of the final state events, whose accuracy is challenged by the outstanding precision of experimental measurements. In this Letter we present the first matched computation of top-quark pair production at next-to-next-to-leading order in QCD with all-order radiative corrections as implemented via parton-shower simulations. Besides its intrinsic relevance for LHC phenomenology, this work also establishes an important step towards the simulation of other hadronic processes with color charges in the final state.
\end{abstract}

DOI: 10.1103/PhysRevLett.127.062001

Top quarks are the heaviest elementary particles observed in nature and play a unique role in the standard model (SM) of particle physics. The large Yukawa coupling of the top quark to the Higgs boson establishes a special avenue in the exploration of the Higgs sector of the SM [1] and of new physics signals [2]. Moreover, the value of the top-quark mass enters precision electroweak (EW) tests [3] and theoretical considerations on the stability of our Universe [4].

At the Large Hadron Collider (LHC), top quarks are predominantly produced by strong interactions in association with their own antiparticle $(t \bar{t})$. The large value of the top-quark mass is such that its production dynamics is safely inside the region of validity of QCD perturbation theory. Remarkable theoretical advancements in the past years have led to very accurate predictions for this process. Specifically, fixed-order computations that rely on a power expansion in the strong coupling constant $\alpha_{s}$ are known up to next-to-next-to-leading order (NNLO) [5-12] (also including the top-quark decays [13]), and EW corrections have been computed up to next-to-leading order (NLO) [14-18]. In specific kinematic regimes, a reliable perturbative description requires the all-order resummation of large radiative corrections [19-25]. Some of the above calculations have been consistently combined to obtain the state-of-the-art predictions at the LHC [26]. The striking

Published by the American Physical Society under the terms of the Creative Commons Attribution 4.0 International license. Further distribution of this work must maintain attribution to the author(s) and the published article's title, journal citation, and DOI. Funded by SCOAP . accuracy of experimental measurements of the top-quark mass requires pushing theoretical calculations to the edge of what can be achieved with perturbative methods (for recent reviews see Refs. [27,28]), and motivates new studies of nonperturbative aspects of top-quark physics (see, e.g., Refs. [29-32]).

The large number of top-quark pairs produced at the LHC has allowed for very satisfactory tests of the theory, both for the inclusive production cross section and for multidifferential distributions [33-41]. These tests have paved the way to the exploitation of top cross-section measurements for the extraction of fundamental parameters of the standard model, such as $\alpha_{s}$ and parton density functions (PDFs), the top mass itself, and the top Yukawa couplings (see, e.g., Refs. [42-48]).

Experimental analyses involving $t \bar{t}$ production heavily rely upon its fully exclusive simulation. This is important not only for the study of the production dynamics itself, but, due to the complex final states that involve a combination of leptons, jets, $b$ hadrons, and missing energy, also for several SM processes and new physics searches for which the $t \bar{t}$ process acts as background. The needed simulations rely on event generators, which combine a prediction for the high-energy scattering, which produces the $t \bar{t}$ pair, initial- and final-state QCD radiation at all perturbative orders via parton shower (PS) algorithms and hadronization models (for a review see Ref. [49]). These event generators have been the subject of considerable research, dealing with the matching of NLO QCD calculations to PS and a consistent description of the top-quark resonance [50-55]. Current research for the improvement of event generators focuses upon the development of more 
accurate PS [56-62], as well as a framework to combine NNLO computations with PS into a consistent event generator (NNLO + PS in the following).

Different frameworks for NNLO + PS computations have been developed in recent years in the context of color-singlet production [63-67]. However, nearly a decade after these developments, a NNLO + PS method to deal with hadron-collider processes with color charges in the final state (e.g., $t \bar{t}$ ), which are considerably more complex, is still missing.

In this Letter, we show how a generator of the same type as the ones developed for color-singlet production processes in Refs. [66,67], dubbed there MINNLO $_{\mathrm{PS}}$, can be constructed for top-quark pair production. Our work constitutes the first computation of this type for reactions with colored particles in the final state.

The MINNLO ${ }_{\mathrm{PS}}$ procedure involves three steps. The first one (referred to as Step I in the following) corresponds to the generation of a $t \bar{t}$ pair plus one light parton (i.e., the underlying Born configuration) according to the POWHEG method [68-71], carried out at the NLO level, inclusive over the radiation of a second light parton.

The second step (step II) characterizes the MINNLO ${ }_{\mathrm{PS}}$ procedure, and it concerns the limit in which the light partons in the above calculation become unresolved (i.e., the underlying Born degenerates into a $t \bar{t}$ configuration without light jets). In this limit the calculation must be supplemented with an appropriate Sudakov form factor and higher-order terms, so as to guarantee that the simulation remains finite as well as NNLO accurate for inclusive $t \bar{t}$ production. Most of the novelties in this Letter have to do with step II and will be illustrated below.

In the third step (step III), the kinematics of the second radiated parton, accounted for inclusively in step I, is generated according to the standard POWHEG method, which guarantees that the NLO accuracy of the $t \bar{t}+$ jet cross section is preserved. From this point on, subsequent radiation is included by the parton shower, with the constraint of having a transverse momentum softer than that of the last POWHEG real emission.

The starting point to achieve NNLO accuracy in step II is the well-known factorization theorem for $t \bar{t}$ pair production at small transverse momentum $p_{T} \equiv\left|\vec{p}_{T}\right|$ differential in the phase space of the $t \bar{t}$ pair $d \Phi_{t \bar{t}} \equiv d \bar{x}_{1} d \bar{x}_{2}\left[d \Phi_{2}\right]$. Here $\bar{x}_{1,2}=m_{t \bar{t}} / \sqrt{s} e^{ \pm y_{t \bar{t}}}$, with $y_{t \bar{t}}$ being the rapidity of the $t \bar{t}$ system, $m_{t \bar{t}}$ its invariant mass, $\left[d \Phi_{2}\right]$ denotes the Lorentzinvariant two-body phase space, and $\sqrt{s}$ is the collider center-of-mass energy. It reads [19-22]

$$
\begin{aligned}
\frac{d \sigma}{d^{2} \vec{p}_{T} d \Phi_{\bar{t} \bar{t}}}= & \sum_{c=q, \bar{q}, g} \frac{\left|M_{c \bar{c}}^{(0)}\right|^{2}}{2 m_{t \bar{t}}^{2}} \int \frac{d^{2} \vec{b}}{(2 \pi)^{2}} e^{i \vec{b} \cdot \vec{p}_{T}} e^{-S_{c}\left(\frac{b_{0}}{b}\right)} \\
& \times \sum_{i, j} \operatorname{Tr}\left(\mathbf{H}_{c} \boldsymbol{\Delta}\right)\left(C_{c i} \otimes f_{i}\right)\left(C_{\bar{c} j} \otimes f_{j}\right),
\end{aligned}
$$

where $b_{0}=2 e^{-\gamma_{E}}, b=|\vec{b}| . S_{c}$ is the Sudakov radiator which also enters the description of the production of a color-singlet system at small transverse momentum

$$
S_{c}(k)=\int_{k^{2}}^{m_{t t}^{2}} \frac{d q^{2}}{q^{2}}\left[A\left(\alpha_{s}(q)\right) \ln \frac{m_{t \bar{t}}^{2}}{q^{2}}+B\left(\alpha_{s}(q)\right)\right] .
$$

The first sum in Eq. (1) runs over all possible flavor configurations of the incoming partons $p_{1}$ of flavor $c$ and $p_{2}$ of flavor $\bar{c}$. The collinear coefficient functions $C_{i j}=$ $C_{i j}\left(z, p_{1}, p_{2}, \vec{b} ; \alpha_{s}\left(b_{0} / b\right)\right)$ describe the structure of constant terms related to the emission of collinear radiation, and the parton densities are denoted by $f_{i}$ and are evaluated at $b_{0} / b$. The operation $\otimes$ denotes the standard convolution over the momentum fraction $z$ carried by initial state radiation. The factor $\operatorname{Tr}\left(\mathbf{H}_{c} \boldsymbol{\Delta}\right)\left(C_{c i} \otimes f_{i}\right)\left(C_{\bar{c} j} \otimes f_{j}\right)$ has different expressions for the $q \bar{q}$ and $g g$ channels and has here a symbolic meaning. In particular, it has a rich Lorentz structure that we omit for simplicity in Eq. (1), which is a source of azimuthal correlations in the collinear limit [21,72].

All quantities in bold face denote operators in color space, and the trace $\operatorname{Tr}\left(\mathbf{H}_{c} \boldsymbol{\Delta}\right)$ in Eq. (1) runs over the color indices. This term can be expressed conveniently in the color space formalism of Ref. [73], where the infraredsubtracted amplitude $\left|M_{c \bar{c}}\right\rangle$ for the production of the $t \bar{t}$ system is a vector in color space $[12,21]$. It reads

$$
\operatorname{Tr}\left(\mathbf{H}_{c} \boldsymbol{\Delta}\right)=\frac{\left\langle M_{c \bar{c}}|\mathbf{\Delta}| M_{c \bar{c}}\right\rangle}{\left|M_{c \bar{c}}^{(0)}\right|^{2}},
$$

where $\left|M_{c \bar{c}}^{(0)}\right|^{2}=\left\langle M_{c \bar{c}}^{(0)} \mid M_{c \bar{c}}^{(0)}\right\rangle$. The hard function $\mathbf{H}_{c}=$ $\mathbf{H}_{c}\left(\Phi_{\bar{t}} ; \alpha_{s}\left(m_{i \bar{t}}\right)\right)$ is obtained from the subtracted amplitudes and the ambiguity in its definition corresponds to using a specific resummation scheme [74]. We adopt here the definition of Ref. [21]. The operator $\Delta$ encodes the structure of the quantum interference due to the exchange of soft radiation at large angle between the initial and final state, and within the final state. It is given by $\boldsymbol{\Delta}=\mathbf{V}^{\dagger} \mathbf{D V}$, where [21]

$$
\mathbf{V}=\mathcal{P} \exp \left\{-\int_{b_{0}^{2} / b^{2}}^{m_{t \hbar}^{2}} \frac{d q^{2}}{q^{2}} \boldsymbol{\Gamma}_{t}\left(\Phi_{t \bar{t}} ; \alpha_{s}(q)\right)\right\} .
$$

The symbol $\mathcal{P}$ denotes the path ordering (with increasing scales from left to right) of the exponential matrix with respect to the integration variable $q^{2}$. $\boldsymbol{\Gamma}_{t}$ is the anomalous dimension accounting for the effect of real soft radiation at large angles, and $\mathbf{D}=\mathbf{D}\left(\Phi_{t \bar{t}}, \vec{b} ; \alpha_{s}\left(b_{0} / b\right)\right)$ encodes the azimuthal dependence of the corresponding constant terms, and is defined such that $[\mathbf{D}]_{\phi}=\mathbb{1}$, where $[\cdots]_{\phi}$ denotes the average over the azimuthal angle $\phi$ of $\vec{p}_{T}$.

All of the above quantities admit a perturbative expansion in a series in $\alpha_{s}(\mu) /(2 \pi)$, where $\mu$ is the scale indicated explicitly in the argument of each function. We generically denote these expansions as 


$$
F\left(\{x\} ; \alpha_{s}(\mu)\right)=\sum_{i} \frac{\alpha_{s}^{i}(\mu)}{(2 \pi)^{i}} F^{(i)}(\{x\}),
$$

with $\{x\}$ being any other set of arguments of $F \equiv\left\{A, B, C_{i j}, \mathbf{H}_{c}, \mathbf{D}, \boldsymbol{\Gamma}_{t},\left|M_{c \bar{c}}\right\rangle\right\}$. We do not indicate explicitly the scale of the amplitude $\left|M_{c \bar{c}}\right\rangle$. In this case, the expansion (5) is in powers of $\alpha_{s}\left(m_{t \bar{t}}\right)$, and each of its perturbative coefficients $\left|M_{c \bar{c}}^{(i)}\right\rangle$ includes an extra single power of $\alpha_{s}\left(\mu_{R}^{(0)}\right)$ with $\mu_{R}^{(0)} \sim m_{t \bar{t}}$. The above coefficients $F^{(i)}$ up to two loops are given in Refs. [12,20,21,72,75-86]. The two-loop coefficient $\mathbf{D}^{(2)}$ is irrelevant at NNLO for observables averaged over $\phi$, since $[\mathbf{D}]_{\phi}=\mathbb{1}$, and therefore we do not include it here.

Expanding the second order term in the exponential of Eq. (4), one can write

$$
\begin{aligned}
\mathbf{V}= & \mathcal{P}\left[\exp \left\{-\int_{b_{0}^{2} / b^{2}}^{m_{t \hbar}^{2}} \frac{d q^{2}}{q^{2}} \frac{\alpha_{s}(q)}{2 \pi} \boldsymbol{\Gamma}_{t}^{(1)}\right\}\right. \\
& \left.\times\left(1-\int_{b_{0}^{2} / b^{2}}^{m_{t \grave{t}}^{2}} \frac{d q^{2}}{q^{2}} \frac{\alpha_{s}^{2}(q)}{(2 \pi)^{2}} \boldsymbol{\Gamma}_{t}^{(2)}\right)\right]+\mathcal{O}\left(\mathrm{N}^{3} \mathrm{LL}\right),
\end{aligned}
$$

where we neglected $\mathrm{N}^{3} \mathrm{LL}$ corrections which do not contribute at NNLO in Eq. (1). In the following we will denote by $\mathbf{V}_{\mathrm{NLL}}$ the right-hand side of Eq. (6) with $\boldsymbol{\Gamma}_{t}^{(2)} \rightarrow 0$. This enters the description of the $p_{T}$ spectrum at NLL accuracy.

To make contact with the procedure described in Ref. [66] we need to simplify further the structure of the term $\mathbf{H}_{c} \boldsymbol{\Delta}$, which encodes the difference with the color singlet case. Our goal is to obtain a closed formula in $p_{T}$ space that retains NNLO accuracy. To this order, we observe that we can take the $\boldsymbol{\Gamma}_{t}^{(2)}$ term in Eq. (6) out of the path ordering symbol. We then perform a rotation in color space to diagonalize $\boldsymbol{\Gamma}_{t}^{(1)}$ and evaluate the exponential matrix in Eq. (6). Equation (1) can be reorganized using

$$
\begin{aligned}
e^{-S_{c}\left(\frac{b_{0}}{b}\right)} \operatorname{Tr}\left(\mathbf{H}_{c} \mathbf{\Delta}\right)= & e^{-\hat{S}_{c}\left(\frac{b_{0}}{b}\right)} \frac{\left\langle M_{c \bar{c}}^{(0)}\left|\left(\mathbf{V}_{\mathrm{NLL}}\right)^{\dagger} \mathbf{V}_{\mathrm{NLL}}\right| M_{c \bar{c}}^{(0)}\right\rangle}{\left|M_{c \bar{c}}^{(0)}\right|^{2}} \\
& \times \operatorname{Tr}\left(\mathbf{H}_{c} \mathbf{D}\right)+E\left(\Phi_{\bar{t} \bar{t}}, \vec{b}\right)+\mathcal{O}\left(\alpha_{s}^{5}\right),
\end{aligned}
$$

where the trace is to be interpreted as in Eq. (3). The Sudakov radiator $\hat{S}_{c}$ is obtained from $S_{c}$ via the replacement

$$
\begin{aligned}
B^{(2)} & \rightarrow B^{(2)}+\frac{\left\langle M_{c \bar{c}}^{(0)}\left|\boldsymbol{\Gamma}_{t}^{(2) \dagger}+\boldsymbol{\Gamma}_{t}^{(2)}\right| M_{c \bar{c}}^{(0)}\right\rangle}{\left|M_{c \bar{c}}^{(0)}\right|^{2}} \\
+ & \frac{1}{\left|M_{c \bar{c}}^{(0)}\right|^{2}} 2 \Re\left[\left\langle M_{c \bar{c}}^{(1)}\left|\boldsymbol{\Gamma}_{t}^{(1) \dagger}+\boldsymbol{\Gamma}_{t}^{(1)}\right| M_{c \bar{c}}^{(0)}\right\rangle\right] \\
- & 2 \frac{\left\langle M_{c \bar{c}}^{(0)}\left|\boldsymbol{\Gamma}_{t}^{(1) \dagger}+\boldsymbol{\Gamma}_{t}^{(1)}\right| M_{c \bar{c}}^{(0)}\right\rangle}{\left|M_{c \bar{c}}^{(0)}\right|^{4}} \Re\left[\left\langle M_{c \bar{c}}^{(1)} \mid M_{c \bar{c}}^{(0)}\right\rangle\right] .
\end{aligned}
$$

The remainder term $E\left(\Phi_{i \bar{t}}, \vec{b}\right)$ in Eq. (7) contributes at order $\alpha_{s}^{2} \ln \left(m_{t \bar{t}} b\right)$, but it is irrelevant for our computation since it vanishes upon azimuthal integration (i.e., $[E]_{\phi}=0$ ). For this reason, we will ignore it in the following. We then obtain

$$
\begin{aligned}
\frac{d \sigma}{d^{2} \vec{p}_{T} d \Phi_{t \bar{t}}}= & \frac{1}{2 m_{t \bar{t}}^{2}} \sum_{c=q, \bar{q}, g} \int \frac{d^{2} \vec{b}}{(2 \pi)^{2}} e^{i \vec{b} \cdot \vec{p}_{T}} e^{-\hat{S}_{c}\left(\frac{b_{0}}{b}\right)} \\
& \times\left\langle M_{c \bar{c}}^{(0)}\left|\left(\mathbf{V}_{\mathrm{NLL}}\right)^{\dagger} \mathbf{V}_{\mathrm{NLL}}\right| M_{c \bar{c}}^{(0)}\right\rangle \\
& \times \sum_{i, j} \operatorname{Tr}\left(\mathbf{H}_{c} \mathbf{D}\right)\left(C_{c i} \otimes f_{i}\right)\left(C_{\bar{c} j} \otimes f_{j}\right)+\mathcal{O}\left(\alpha_{s}^{5}\right) .
\end{aligned}
$$

This expression has almost the structure needed in order to carry out the same procedure used in the color singlet case $[66,67]$, except for the $\mathbf{H}_{c}$ function which needs to be evaluated at the scale $b_{0} / b$ rather than $m_{t \bar{t}}$. To the relevant accuracy we can perform this scale change provided $B^{(2)}$ in $\hat{S}_{c}$ is also modified as follows $[63,66]$ [see, e.g., Eq. (4.25) of Ref. [66] ]

$$
B^{(2)} \rightarrow B^{(2)}+2 \pi \beta_{0} \frac{2 \Re\left[\left\langle M_{c \bar{c}}^{(1)} \mid M_{c \bar{c}}^{(0)}\right\rangle\right]}{\left|M_{c \bar{c}}^{(0)}\right|^{2}} .
$$

In the color basis in which $\boldsymbol{\Gamma}_{t}^{(1)}$ is diagonal, the matrix element $\left\langle M_{c \bar{c}}^{(0)}\left|\left(\mathbf{V}_{\mathrm{NLL}}\right)^{\dagger} \mathbf{V}_{\mathrm{NLL}}\right| M_{c \bar{c}}^{(0)}\right\rangle$ is a linear combination of complex exponential terms, each of which has the same factorized structure used as a starting point in the appendix of Ref. [66].

Using this observation, we finally integrate over $\vec{b}$ by expanding the integrand about $b_{0} / b \sim p_{T}$. Noticing that the matrix element $\left\langle M_{c \bar{c}}^{(0)}\left|\left(\mathbf{V}_{\mathrm{NLL}}\right)^{\dagger} \mathbf{V}_{\mathrm{NLL}}\right| M_{c \bar{c}}^{(0)}\right\rangle$ does not depend on the azimuthal angle $\phi$, up to terms of $\mathcal{O}\left(\alpha_{s}^{5}\right)$ we can express the result as a total derivative, leading to the final $p_{T}$ space formula

$$
\begin{aligned}
\frac{d \sigma}{d p_{T} d \Phi_{t \bar{t}}}= & \frac{d}{d p_{T}}\left\{\sum_{c} \frac{e^{-\tilde{S}_{c}\left(p_{T}\right)}}{2 m_{t \bar{t}}^{2}}\left\langle M_{c \bar{c}}^{(0)}\left|\left(\mathbf{V}_{\mathrm{NLL}}\right)^{\dagger} \mathbf{V}_{\mathrm{NLL}}\right| M_{c \bar{c}}^{(0)}\right\rangle\right. \\
& \left.\times \sum_{i, j}\left[\operatorname{Tr}\left(\tilde{\mathbf{H}}_{c} \mathbf{D}\right)\left(\tilde{C}_{c i} \otimes f_{i}\right)\left(\tilde{C}_{\bar{c} j} \otimes f_{j}\right)\right]_{\phi}\right\} \\
& +R_{f}+\mathcal{O}\left(\alpha_{s}^{5}\right) .
\end{aligned}
$$

To obtain Eq. (11), we introduced the quantities $\tilde{S}_{c}, \tilde{\mathbf{H}}_{c}$, and $\tilde{C}_{i j}$, which are obtained by applying the transformations given in Eq. (4.24) of Ref. [66] to $\hat{S}_{c}, \mathbf{H}_{c}$, and $C_{i j}$. The latter quantities are now evaluated at the scale $p_{T}$. The azimuthally averaged term $[\cdots]_{\phi}$ in Eq. (11) is taken from the NNLO computation of the $t \bar{t}$ cross section of Refs. [11,12,86] (see also Ref. [87] for more details). We also included the remainder $R_{f}=R_{f}\left(p_{T}\right)$, which 
denotes the regular contribution to the $p_{T}$ distribution through $\mathcal{O}\left(\alpha_{s}^{4}\right)$, such that $p_{T} R_{f}\left(p_{T}\right)$ vanishes in the $p_{T} \rightarrow$ 0 limit. The integral of Eq. (11) over $p_{T}$ provides a NNLO accurate description of $t \bar{t}$ production differential in $\Phi_{t \bar{t}}$.

In order to build a Monte Carlo algorithm for the generation of events with NNLO accuracy, we have to modify the formula for the underlying Born cross section of step I in such a way that it matches Eq. (11) maintaining its NNLO accuracy. The procedure is described in detail in Refs. $[63,66,67,88]$ and for this reason we omit it here. For the practical implementation, we use the NLO + PS code for $t \bar{t}+$ jet of Ref. [89], and apply the MINNLO ${ }_{\mathrm{PS}}$ procedure for heavy-quark pair production given in this Letter. The PS simulation is obtained with PYTHIA8 [90], without the modeling of nonperturbative effects, and under the assumption of stable top quarks. We stress that up to Eq. (10) we retained also NLL accuracy in the $p_{T}$ spectrum, while Eq. (11) is strictly LL accurate. Higher logarithmic accuracy could, in principle, be maintained in Eq. (11). However, this higher accuracy would be spoiled by the PS used here, which is limited to LL. On the other hand, Eq. (11) also preserves the class of NLL corrections associated with the coefficient $A^{(2)}$ in the Sudakov, which are traditionally included in PS algorithms [91]. The formulation of a $(\mathrm{N}) \mathrm{NLO}$ matching to PS that preserves logarithmic accuracy beyond LL is still an open problem.

In the phenomenological study presented below, we consider LHC collisions with a center-of-mass energy of $13 \mathrm{TeV}$. The top-quark pole mass is set to $173.3 \mathrm{GeV}$ and we consider five massless quark flavors using the corresponding NNLO set of the NNPDF31 [92] parton densities with $\alpha_{s}\left(m_{Z}\right)=0.118$. The renormalization scale for the two powers of the strong coupling constant entering the Born cross section is set to $\mu_{R}^{(0)}=K_{R} m_{t \bar{t}} / 2$. In the rest of Eq. (11), we implement the renormalization $\left(\mu_{R}=K_{R} \mu_{0}\right)$ and factorization $\left(\mu_{F}=K_{F} \mu_{0}\right)$ scale dependence as described in Ref. [66], with the central scale $\mu_{0}=$ $m_{t \bar{t}} / 2 e^{-L}$ [hence replacing the scales set to $p_{T}$ in Eq. (11)], where we defined $L=\ln Q / p_{T}$ and $Q=m_{t \bar{t}} / 2$. The logarithm $L$ is turned off in the hard region of the $p_{T}$ spectrum so that the total derivative in Eq. (11) smoothly vanishes for $p_{T} \gtrsim Q$ as in Refs. [74,9396]. The dependence of Eq. (11) on $\mu_{R}, \mu_{F}$, and $Q$ is of order $\mathcal{O}\left(\alpha_{s}^{5}\right)$. At small $p_{T}$ the scale of the strong coupling and the parton densities is smoothly frozen around $Q_{0}=$ $2 \mathrm{GeV}$ following the procedure of Ref. [67] to avoid the Landau singularity. To estimate the scale uncertainties we vary $K_{R}$ and $K_{F}$ by a factor of 2 around their central value, while keeping $1 / 2 \leq K_{R} / K_{F} \leq 2$. Results with a different central scale choice are reported in Supplemental Material [97].

For comparison, we consider results from the fixed-order NNLO calculation of Refs. [11,12] obtained with the MATRIX code [98] using $\mu_{0}=m_{t \bar{t}} / 2$. Furthermore, we also show MINLO' results, obtained with the NLO + PS
TABLE I. The total $t \bar{t}$ cross section in different approximations. The quoted errors represent the scale uncertainty, while the numbers in brackets are the numerical uncertainty on the last digit.

\begin{tabular}{ccc}
\hline \hline MINLO $^{\prime}$ & NNLO & MINNLO $_{\text {PS }}$ \\
\hline $695.6(3)_{-17 \%}^{+22 \%} \mathrm{pb}$ & $769.8(9)_{-6.5 \%}^{+5.0 \%} \mathrm{pb}$ & $775.5(2)_{-7.2 \%}^{+9.8 \%} \mathrm{pb}$ \\
\hline \hline
\end{tabular}

generator for $t \bar{t}$ plus zero and one jet, constructed by turning off the NNLO corrections in Eq. (11). The latter constitutes a new calculation as well.

Table I shows the total cross section for top-quark pair production for MINLO', NNLO, and MINNLO ${ }_{\mathrm{PS}}$. The central MINLO' result is about $10.3 \%(9.6 \%)$ smaller than the MINNLO $_{\text {PS }}$ (NNLO) prediction and features much larger scale uncertainties. The MINNLO $_{\mathrm{PS}}$ result instead agrees with NNLO at the subpercent level, well within the perturbative uncertainties. Small numerical differences are expected even for inclusive observables, since the MINNLO $_{P S}$ and NNLO calculations differ by terms beyond accuracy.

In Fig. 1 we examine a set of differential distributions. To validate MINNLO $_{\mathrm{PS}}$, we compare it to the NNLO prediction without fiducial cuts, which could lead to significant differences due to the PS. Experimental data from the CMS Collaboration unfolded and extrapolated to the inclusive phase space [99], and divided by the appropriate branching fractions, are also shown. The top-left plot shows the rapidity difference between the $t \bar{t}$ system and the leading jet defined with $p_{T, j_{1}} \geq 120 \mathrm{GeV}$. Both MINLO' and MINNLO $_{\mathrm{PS}}$ are formally NLO accurate in this case, and the agreement between them indicates that this accuracy is retained by the MINNLO PS $_{\text {procedure. The same }}$ conclusion holds for other observables that require at least one resolved hard jet.

The distributions in the average top-quark rapidity $\left(y_{t_{\mathrm{av}}}\right)$ and transverse momentum $\left(p_{T, t_{\mathrm{av}}}\right)$ as well as in the invariant mass $\left(m_{t \bar{t}}\right)$ and rapidity $\left(y_{t \bar{t}}\right)$ of the $t \bar{t}$ pair shown in Fig. 1 are inclusive over QCD radiation. For such distributions MINNLO $_{\mathrm{PS}}$ is expected to be NNLO accurate. Indeed, MINNLO $_{\mathrm{PS}}$ and NNLO yield consistent results, with fully overlapping uncertainty bands. The small differences in the central value are once again due to the different treatment of terms beyond NNLO accuracy. The larger uncertainty

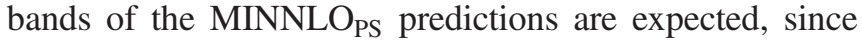
additional scale dependent terms are included within the first term in the right-hand side of Eq. (11) that are not present in the fixed-order calculation. In comparison to the $\mathrm{MINLO}^{\prime}$ results the inclusion of NNLO corrections through MINNLO $_{\mathrm{PS}}$ has an impact of about $10 \%-20 \%$ on the differential distributions and substantially reduces the perturbative uncertainties. Also the agreement with data is quite remarkable. All data points are within 1 standard deviation from the MINNLO $_{\mathrm{PS}}$ prediction, with the 

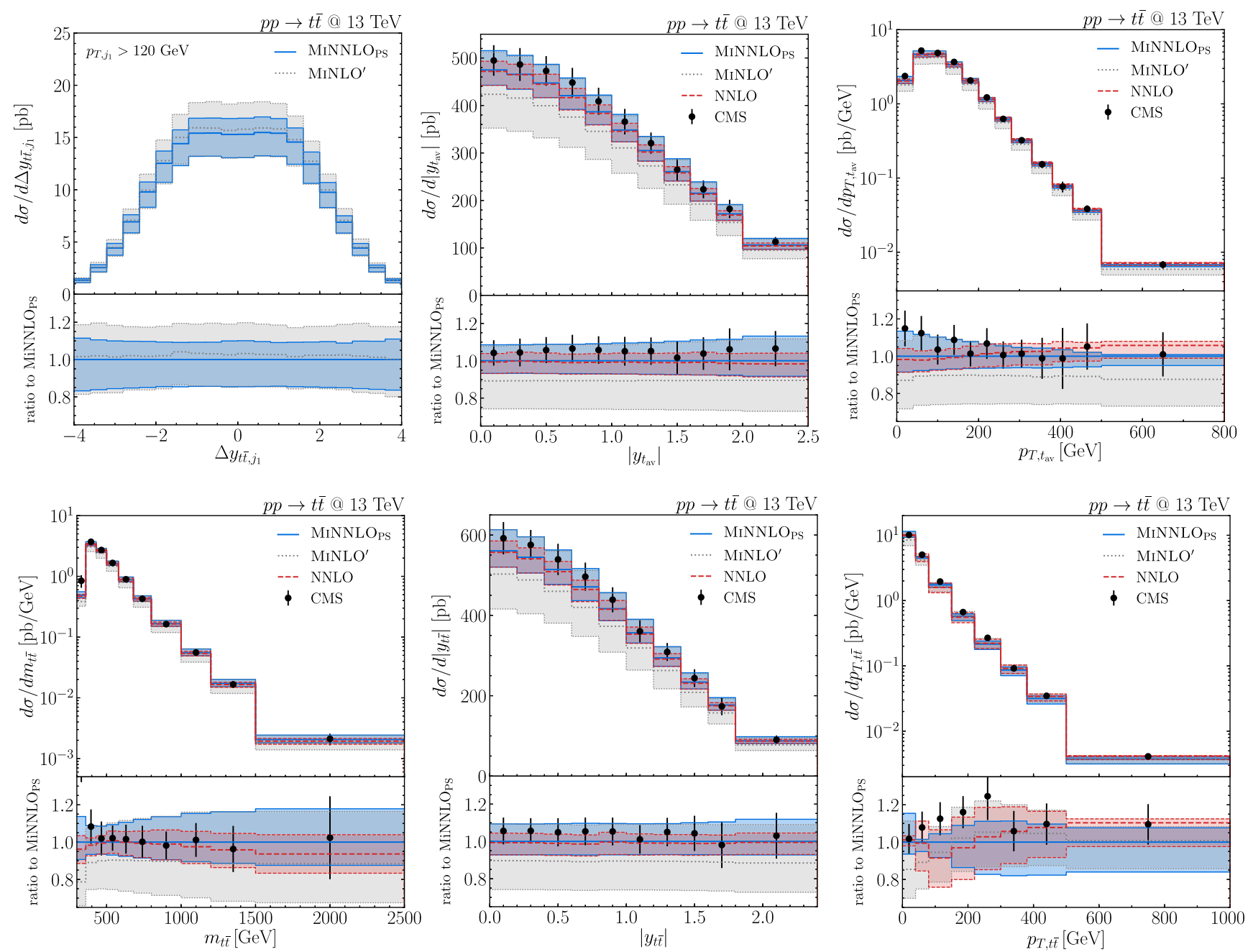

FIG. 1. Distribution in the rapidity difference between the $t \bar{t}$ pair and the leading jet $\left(\Delta y_{t \bar{t}, j_{1}}\right)$, in the rapidity ( $\left.y_{t_{\mathrm{av}}}\right)$ and the average transverse-momentum $\left(p_{T, t_{\mathrm{av}}}\right)$ of the top and the antitop, as well as in the rapidity $\left(y_{t \bar{t}}\right)$, in the invariant mass $\left(m_{t \bar{t}}\right)$ and in the transverse

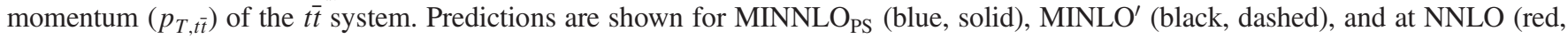
dashed). The black data points represent the CMS measurement at $13 \mathrm{TeV}$ of Ref. [99], where the $y_{t_{\mathrm{av}}}$ and $p_{T, t_{\mathrm{av}}}$ distributions have been obtained with leptonically decaying top quarks.

exception of the very first bin in the $m_{t \bar{t}}$ distribution that, on the other hand, is strongly affected by the finite width of the top, whose effects are not included here.

We finally discuss the transverse-momentum spectrum of the $t \bar{t}$ pair, denoted by $p_{T, t \bar{t}}$ in the bottom-right panel of Fig. 1. At large transverse momenta, the three predictions considered are effectively NLO accurate. Indeed, MINLO' and MINNLO $_{\mathrm{PS}}$ are essentially indistinguishable in that region, and at the same time consistent with the spectrum at fixed order. The small differences with NNLO are due to the generation of further radiation by the PS. At small transverse momenta, MINNLO ${ }_{\mathrm{PS}}$ induces $\mathcal{O}(10 \%)$ corrections with respect to MINLO $^{\prime}$ and significantly reduces the large scale dependence. In this region, it also differs in shape from the NNLO calculation, which diverges and becomes unphysical for vanishing transverse momenta. Within the relatively large experimental errors, MINNLO $_{P S}$ slightly improves the description of the data in terms of shape compared to NNLO for this observable.

In this Letter we have presented the matching of the NNLO computation for top-quark pair production at hadron colliders with parton showers. This result has been obtained by constructing the MINNLO ${ }_{\mathrm{PS}}$ method for the production of heavy quarks, which constitutes the first $\mathrm{NNLO}+\mathrm{PS}$ prediction for reactions with color charges in the final state in hadronic collisions. The comparisons presented in Fig. 1 provide a numerical validation of MINNLO $_{\mathrm{PS}}$ for top-quark pair production, demonstrating its NNLO accuracy. The simulations presented here also allow for the inclusion of the top-quark decay, paving the way to an accurate event generation for $t \bar{t}$ production at the 
LHC which will enable precise comparisons of fiducial measurements to theory.

We would like to thank Stefano Catani, Massimiliano Grazzini, and Keith Hamilton for discussions and comments on the manuscript. P. N. acknowledges support from Fondazione Cariplo and Regione Lombardia, Grant No. 2017-2070, and from INFN.

[1] D. de Florian et al. (LHC Higgs Cross Section Working Group), arXiv:1610.07922.

[2] I. Brivio, S. Bruggisser, F. Maltoni, R. Moutafis, T. Plehn, E. Vryonidou, S. Westhoff, and C. Zhang, J. High Energy Phys. 02 (2020) 131.

[3] M. Baak, J. Cúth, J. Haller, A. Hoecker, R. Kogler, K. Mönig, M. Schott, and J. Stelzer (Gfitter Group Collaboration), Eur. Phys. J. C 74, 3046 (2014).

[4] G. Degrassi, S. Di Vita, J. Elias-Miro, J. R. Espinosa, G. F. Giudice, G. Isidori, and A. Strumia, J. High Energy Phys. 08 (2012) 098.

[5] P. Bärnreuther, M. Czakon, and A. Mitov, Phys. Rev. Lett. 109, 132001 (2012).

[6] M. Czakon and A. Mitov, J. High Energy Phys. 12 (2012) 054.

[7] M. Czakon and A. Mitov, J. High Energy Phys. 01 (2013) 080.

[8] M. Czakon, P. Fiedler, and A. Mitov, Phys. Rev. Lett. 110, 252004 (2013).

[9] M. Czakon, D. Heymes, and A. Mitov, Phys. Rev. Lett. 116, 082003 (2016).

[10] M. Czakon, P. Fiedler, D. Heymes, and A. Mitov, J. High Energy Phys. 05 (2016) 034.

[11] S. Catani, S. Devoto, M. Grazzini, S. Kallweit, J. Mazzitelli, and H. Sargsyan, Phys. Rev. D 99, 051501(R) (2019).

[12] S. Catani, S. Devoto, M. Grazzini, S. Kallweit, and J. Mazzitelli, J. High Energy Phys. 07 (2019) 100.

[13] A. Behring, M. Czakon, A. Mitov, R. Poncelet, and A. S. Papanastasiou, Phys. Rev. Lett. 123, 082001 (2019).

[14] W. Beenakker, A. Denner, W. Hollik, R. Mertig, T. Sack, and D. Wackeroth, Nucl. Phys. B411, 343 (1994).

[15] W. Bernreuther, M. Fücker, and Z. Si, Phys. Lett. B 633, 54 (2006); 644, 386(E) (2007).

[16] J. H. Kuhn, A. Scharf, and P. Uwer, Eur. Phys. J. C 51, 37 (2007).

[17] A. Denner and M. Pellen, J. High Energy Phys. 08 (2016) 155.

[18] M. Czakon, D. Heymes, A. Mitov, D. Pagani, I. Tsinikos, and M. Zaro, J. High Energy Phys. 10 (2017) 186.

[19] H. X. Zhu, C. S. Li, H. T. Li, D. Y. Shao, and L. L. Yang, Phys. Rev. Lett. 110, 082001 (2013).

[20] H. T. Li, C. S. Li, D. Y. Shao, L. L. Yang, and H. X. Zhu, Phys. Rev. D 88, 074004 (2013).

[21] S. Catani, M. Grazzini, and A. Torre, Nucl. Phys. B890, 518 (2014).

[22] S. Catani, M. Grazzini, and H. Sargsyan, J. High Energy Phys. 11 (2018) 061.

[23] M. Beneke, P. Falgari, S. Klein, and C. Schwinn, Nucl. Phys. B855, 695 (2012).
[24] M. Beneke, P. Falgari, S. Klein, J. Piclum, C. Schwinn, M. Ubiali, and F. Yan, J. High Energy Phys. 07 (2012) 194.

[25] W.-L. Ju, G. Wang, X. Wang, X. Xu, Y. Xu, and L. L. Yang, J. High Energy Phys. 06 (2020) 158.

[26] M. L. Czakon et al., Chin. Phys. C 44, 083104 (2020).

[27] P. Nason, The top mass in hadronic collisions, in From My Vast Repertoire...: Guido Altarelli's Legacy, edited by A. Levy, S. Forte, and G. Ridolfi (World Scientific, Singapore, 2019), pp. 123-151, https://www.worldscientific.com/doi/ abs/10.1142/9789813238053_0008.

[28] A. H. Hoang, Annu. Rev. Nucl. Part. Sci. 70, 225 (2020).

[29] M. Beneke, P. Marquard, P. Nason, and M. Steinhauser, Phys. Lett. B 775, 63 (2017).

[30] A. H. Hoang, C. Lepenik, and M. Preisser, J. High Energy Phys. 09 (2017) 099.

[31] A. H. Hoang, S. Plätzer, and D. Samitz, J. High Energy Phys. 10 (2018) 200.

[32] S. Ferrario Ravasio, P. Nason, and C. Oleari, J. High Energy Phys. 01 (2019) 203.

[33] G. Aad et al. (ATLAS Collaboration), Phys. Lett. B 810, 135797 (2020).

[34] G. Aad et al. (ATLAS Collaboration), J. High Energy Phys. 01 (2021) 033.

[35] G. Aad et al. (ATLAS Collaboration), Eur. Phys. J. C 80, 528 (2020).

[36] G. Aad et al. (ATLAS Collaboration), Eur. Phys. J. C 79, 1028 (2019).

[37] V. Khachatryan et al. (CMS Collaboration), Eur. Phys. J. C 77, 172 (2017).

[38] A. M. Sirunyan et al. (CMS Collaboration), J. High Energy Phys. 09 (2017) 051.

[39] A. M. Sirunyan et al. (CMS Collaboration), J. High Energy Phys. 02 (2019) 149.

[40] A. Sirunyan et al. (CMS Collaboration), J. High Energy Phys. 06 (2018) 002.

[41] A. Sirunyan et al. (CMS Collaboration), J. High Energy Phys. 04 (2018) 060.

[42] S. Chatrchyan et al. (CMS Collaboration), Phys. Lett. B 728, 496 (2014); 738, 526(E) (2014).

[43] T. Klijnsma, S. Bethke, G. Dissertori, and G. P. Salam, Eur. Phys. J. C 77, 778 (2017).

[44] A. M. Sirunyan et al. (CMS Collaboration), Eur. Phys. J. C 80, 658 (2020).

[45] G. Aad et al. (ATLAS Collaboration), J. High Energy Phys. 11 (2019) 150.

[46] A. M. Sirunyan et al. (CMS Collaboration), Phys. Rev. D 102, 092013 (2020).

[47] A. M. Sirunyan et al. (CMS Collaboration), Phys. Rev. D 100, 072007 (2019).

[48] A. M. Cooper-Sarkar, M. Czakon, M. A. Lim, A. Mitov, and A. S. Papanastasiou, arXiv:2010.04171.

[49] A. Buckley et al., Phys. Rep. 504, 145 (2011).

[50] R. Frederix and S. Frixione, J. High Energy Phys. 12 (2012) 061.

[51] S. Hoeche, F. Krauss, P. Maierhoefer, S. Pozzorini, M. Schonherr, and F. Siegert, Phys. Lett. B 748, 74 (2015).

[52] K. Cormier, S. Plätzer, C. Reuschle, P. Richardson, and S. Webster, Eur. Phys. J. C 79, 915 (2019).

[53] T. Ježo and P. Nason, J. High Energy Phys. 12 (2015) 065. 
[54] T. Ježo, J. M. Lindert, P. Nason, C. Oleari, and S. Pozzorini, Eur. Phys. J. C 76, 691 (2016).

[55] R. Frederix, S. Frixione, A. S. Papanastasiou, S. Prestel, and P. Torrielli, J. High Energy Phys. 06 (2016) 027.

[56] S. Höche, F. Krauss, and S. Prestel, J. High Energy Phys. 10 (2017) 093.

[57] F. Dulat, S. Höche, and S. Prestel, Phys. Rev. D 98, 074013 (2018).

[58] M. Dasgupta, F. A. Dreyer, K. Hamilton, P. F. Monni, and G. P. Salam, J. High Energy Phys. 09 (2018) 033.

[59] G. Bewick, S. Ferrario Ravasio, P. Richardson, and M. H. Seymour, J. High Energy Phys. 04 (2020) 019.

[60] M. Dasgupta, F. A. Dreyer, K. Hamilton, P. F. Monni, G. P. Salam, and G. Soyez, Phys. Rev. Lett. 125, 052002 (2020).

[61] J. R. Forshaw, J. Holguin, and S. Plätzer, J. High Energy Phys. 09 (2020) 014.

[62] K. Hamilton, R. Medves, G. P. Salam, L. Scyboz, and G. Soyez, arXiv:2011.10054.

[63] K. Hamilton, P. Nason, C. Oleari, and G. Zanderighi, J. High Energy Phys. 05 (2013) 082.

[64] S. Alioli, C. W. Bauer, C. Berggren, F. J. Tackmann, J. R. Walsh, and S. Zuberi, J. High Energy Phys. 06 (2014) 089.

[65] S. Höche, Y. Li, and S. Prestel, Phys. Rev. D 91, 074015 (2015).

[66] P. F. Monni, P. Nason, E. Re, M. Wiesemann, and G. Zanderighi, J. High Energy Phys. 05 (2020) 143.

[67] P. F. Monni, E. Re, and M. Wiesemann, Eur. Phys. J. C 80, 1075 (2020).

[68] P. Nason, J. High Energy Phys. 11 (2004) 040.

[69] P. Nason and G. Ridolfi, J. High Energy Phys. 08 (2006) 077.

[70] S. Frixione, P. Nason, and C. Oleari, J. High Energy Phys. 11 (2007) 070.

[71] S. Alioli, P. Nason, C. Oleari, and E. Re, J. High Energy Phys. 06 (2010) 043.

[72] S. Catani and M. Grazzini, Nucl. Phys. B845, 297 (2011).

[73] S. Catani and M. Seymour, Nucl. Phys. B485, 291 (1997).

[74] G. Bozzi, S. Catani, D. de Florian, and M. Grazzini, Nucl. Phys. B737, 73 (2006).

[75] M. Czakon, Phys. Lett. B 664, 307 (2008).

[76] S. Catani and M. Grazzini, Eur. Phys. J. C 72, 2013 (2012); 72, 2132(E) (2012).

[77] M. Czakon and A. Mitov, Comput. Phys. Commun. 185, 2930 (2014).
[78] S. Catani, L. Cieri, D. de Florian, G. Ferrera, and M. Grazzini, Eur. Phys. J. C 72, 2195 (2012).

[79] T. Gehrmann, T. Lübbert, and L. L. Yang, Phys. Rev. Lett. 109, 242003 (2012).

[80] P. Bärnreuther, M. Czakon, and P. Fiedler, J. High Energy Phys. 02 (2014) 078.

[81] T. Gehrmann, T. Luebbert, and L. L. Yang, J. High Energy Phys. 06 (2014) 155.

[82] M. G. Echevarria, I. Scimemi, and A. Vladimirov, J. High Energy Phys. 09 (2016) 004.

[83] R. Angeles-Martinez, M. Czakon, and S. Sapeta, J. High Energy Phys. 10 (2018) 201.

[84] M.-X. Luo, T.-Z. Yang, H. X. Zhu, and Y. J. Zhu, J. High Energy Phys. 01 (2020) 040.

[85] M.-X. Luo, X. Wang, X. Xu, L. L. Yang, T.-Z. Yang, and H. X. Zhu, J. High Energy Phys. 10 (2019) 083.

[86] S. Catani, S. Devoto, M. Grazzini, and J. Mazzitelli (to be published).

[87] H. Sargsyan, Ph. D thesis, University of Zurich, 2017.

[88] K. Hamilton, P. Nason, and G. Zanderighi, J. High Energy Phys. 10 (2012) 155.

[89] S. Alioli, S.-O. Moch, and P. Uwer, J. High Energy Phys. 01 (2012) 137.

[90] T. Sjostrand and P.Z. Skands, Eur. Phys. J. C 39, 129 (2005).

[91] S. Catani, B. R. Webber, and G. Marchesini, Nucl. Phys. B349, 635 (1991).

[92] R. D. Ball et al. (NNPDF Collaboration), Eur. Phys. J. C 77, 663 (2017).

[93] A. Banfi, G. P. Salam, and G. Zanderighi, J. High Energy Phys. 06 (2012) 159.

[94] A. Banfi, P. F. Monni, G. P. Salam, and G. Zanderighi, Phys. Rev. Lett. 109, 202001 (2012).

[95] P. F. Monni, E. Re, and P. Torrielli, Phys. Rev. Lett. 116, 242001 (2016).

[96] W. Bizon, P. F. Monni, E. Re, L. Rottoli, and P. Torrielli, J. High Energy Phys. 02 (2018) 108.

[97] See Supplemental Material at http://link.aps.org/ supplemental/10.1103/PhysRevLett.127.062001 for results with an alternative scale setting.

[98] M. Grazzini, S. Kallweit, and M. Wiesemann, Eur. Phys. J. C 78, 537 (2018).

[99] A. M. Sirunyan et al. (CMS Collaboration), Phys. Rev. D 97, 112003 (2018). 